\title{
Progressão da espessura corneana do ponto mais fino em direção ao limbo: estudo de uma população normal e de portadores de ceratocone para criação de valores de referência
}

\author{
Corneal thickness progression from the thinnest point to the limbus: study \\ based on a normal and a keratoconus population to create reference values
}

\author{
Allan Luz ${ }^{1}$ \\ Mário Ursulio ${ }^{2}$ \\ Daniel Castañeda ${ }^{3}$ \\ Renato Ambrósio Jr. ${ }^{4}$
}

\footnotetext{
Aluno do primeiro ano do Curso de Especialização em Oftalmologia da Fundação Altino Ventura - Recife (PE) - Brasil.

${ }^{2}$ Mestre em Oftalmologia pela Universidade Federal do Rio de Janeiro - UFRJ - Rio de Janeiro (RJ) - Brasil. Diretor do Hospital de Olhos de Sergipe - Aracaju (SE) - Brasil.

${ }^{3}$ Professor de Estatística da Universidade Federal de Sergipe - UFS - Aracaju (SE) - Brasil.

${ }^{4}$ Doutor pelo Departamento de Oftalmologia da Faculdade de Medicina da Universidade de São Paulo - USP São Paulo (SP) - Brasil

Endereço para correspondência: Allan Luz. Fundação Altino Ventura. Rua da Soledade, 170 - Recife (PE) CEP 50070-040

E-mail:allanluz@uol.com.br

Recebido para publicação em 06.10.2005

Versão revisada recebida em 26.12.2005

Aprovação em 03.02.2006

Nota Editorial: Depois de concluída a análise do artigo sob sigilo editorial e com a anuência dos Drs. Fábio Henrique Cacho Casanova e Paulo Gilberto Jorge Fadel sobre a divulgação de seus nomes como revisores, agradecemos sua participação neste processo.
}

\begin{tabular}{l} 
RESUMO \\
\hline Objetivo: Estudar a variação e a progressão dos valores paquimétricos, \\
a partir do ponto mais fino da córnea em direção ao limbo em pacientes \\
normais e com ceratocone; elaborar curvas de referência para esse \\
parâmetro. Métodos: Utilizando-se o mapa paquimétrico numérico do \\
Orbscan, 100 olhos considerados normais e 25 olhos com diagnóstico de \\
ceratocone estágios I e II (Krumeich) foram analisados. Foram demar- \\
cadas circunferências concêntricas no ponto mais fino da córnea, com \\
raios crescentes de 1 a 7 mm. As médias dos pontos de cada circunferên- \\
cia foram calculadas para cada raio e inseridas numa planilha Excel, de \\
modo a possibilitar a criação de um gráfico de progressão para cada caso, \\
a partir do ponto mais fino. O software SPSS foi utilizado para estudos \\
estatísticos. Utilizou-se o teste t de Student como ferramenta para \\
comparação dos valores encontrados. Resultados: A média dos valores \\
no ponto mais fino dos pacientes normais foi igual a 511,60 4 m (desvio \\
padrão de 30,60 ). A média dos valores do ponto mais fino dos pacientes \\
portadores de ceratocone foi 424,48 $\mu$ mo (desvio padrão de 56,57 ). Obser- \\
varam-se diferenças estatisticamente significativas na comparação dos \\
valores médios de todas as circunferências (p<0,01), sendo que para os \\
valores de 6 mm e 7 mm, observou-se p=0,01. Conclusão: Há maior \\
variabilidade de valores paquimétricos nas córneas com ceratocone, bem \\
como há progressão mais acentuada que em olhos normais. A progressão \\
paquimétrica representa uma complementação à propedêutica tradicio- \\
nal da paquimetria pontual central que deve ser considerada no pré- \\
operatório de cirurgias refrativas. Pode também representar índice indi- \\
reto da biomecância do tecido corneano, sendo que essa hipótese merece \\
mais estudos futuros.
\end{tabular}

Descritores: Córnea; Doenças da córnea; Ceratocone; Ceratomileuse assistida por excimer laser in situ; Topografia da córnea; Valores de referência

\section{INTRODUÇÃO}

A espessura da córnea (paquimetria) é um importante parâmetro clínico. Reflete a saúde tecidual, em função de bomba endotelial, sendo fundamental no acompanhamento de pacientes com alterações do endotélio. Além disso, a paquimetria é importante no diagnóstico e acompanhamento de 
580 Progressão da espessura corneana do ponto mais fino em direção ao limbo: estudo de uma população normal e de portadores de ceratocone para criação de valores de referência

doenças ectásicas como ceratocone e degeneração marginal pelúcida. Medidas pontuais centrais são tradicionalmente obtidas com o ultra-som, sendo o parâmetro clínico mais comumente utilizado. Entretanto, mapas paquimétricos, desenvolvidos por sistemas de tomografia de córnea, permitem a determinação do real ponto mais fino e sua localização, bem como avaliar a variação e progressão desses valores na córnea.

A medida da espessura corneana é fundamental em cirurgias refrativas corneanas ${ }^{(1)}$, sendo obrigatória antes da indicação de cirurgia lamelar ou de ablação de superfície. Entretanto o valor central pode não corresponder ao valor mais delgado. Nesse caso, argumenta-se sobre a obrigatoriedade da realização de um mapa paquimétrico.

Adicionalmente, a medida da espessura da córnea é um parâmetro a ser considerado em pacientes com glaucoma, hipertensão ocular ou suspeita de glaucoma. A influência da resistência corneana na medida da pressão intra-ocular (PIO) vem sendo cada vez mais reconhecida ${ }^{(2)}$. O valor da medida da PIO obtido será sub-estimado ou hiper-estimado de acordo com a resistência da córnea ao aplanamento durante a tonometria. De modo geral, assume-se que quanto mais fina é a córnea, menor será a resistência e menor será a medida da pressão - valor sub-estimado. Entretanto, há evidências que a espessura da córnea não reflete a resistência elástica e a flexibilidade do parênquima corneano - biomecância da córnea $^{(3-4)}$. Essas características são ligadas a fatores constitucionais individuais como a composição bioquímica estromal, pelos tipos de colágeno existentes na córnea e quantidade de glocosaminoglicanos (GAGs).

A ectasia iatrogênica é uma complicação rara, mas das mais intrigantes e temidas para o cirurgião refrativo. Ocorre por instabilidade da arquitetura corneana por um leito estromal residual insuficiente para suportar as pressões externas (pressão atmosférica e das pálpebras) e internas (PIO). Há controvérsias sobre qual o leito estromal residual posterior mínimo. Acredita-se que este parâmetro deve ser calculado para cada indivíduo. Barraquer recomendou uma espessura mínima de $300 \mu \mathrm{m}$ para sustentação do estroma corneano, baseado em sua longa experiência pessoal com cirurgia refrativa ${ }^{(5-6)} \mathrm{O}$ FDA (Food and Drug Administration) recomenda que o limite de $250 \mu \mathrm{m}$ não seja violado em procedimentos de $\operatorname{LASIK}^{(7)}$. Desse modo, a paquimetria pré-operatória, a espessura do retalho corneano (flap) e a profundidade de ablação do laser são as variáveis mais importantes. Entretanto, alguns casos de ectasia após LASIK foram descritos com mais de $300 \mu \mathrm{m}$ de leito estromal posterior e sem sinais de doença ectásica na topografia corneana anterior (Placido) no pré-operatório ${ }^{(8-10)}$. Além da possibilidade de um "flap" espesso, a hipótese mais aceita está relacionada com uma predisposição inerente de algumas córneas para instabilidade arquitetônica, de acordo com suas propriedades biomecânicas ${ }^{(3-4)}$. É descrito que a ectasia, com processo progressivo de afinamento e astigmatismo irregular, ocorre rapidamente quando LASIK é realizado em olhos com tal predisposição, identificada topograficamen- te no pré-operatório independentemente, do leito estromal residual $^{(8-14)}$. A maioria dos casos de ectasia não é reportada na literatura, havendo um número relativamente pequeno de casos, havendo uma preocupação sobre uma potencial epidemia desta grave complicação ${ }^{(8-17)}$. Com isso, reconhecemos a necessidade de se aumentar o patamar de segurança para os procedimentos refrativos por meio de uma propedêutica mais sensível para detectar casos com maior risco para esta complicação.

O objetivo deste trabalho é verificar a hipótese da progressão dos valores de espessura corneana como uma característica que pode diferenciar córneas normais de ceratocone. Com isso, foi estudada a variação e progressão dos valores paquimétricos do ponto mais fino da córnea em direção ao limbo, utilizando o mapa paquimétrico do Orbscan, a fim da criação de valores de referência para olhos normais e portadores de ceratocone leve.

\section{MÉTODOS}

Foram estudados de forma retrospectiva 100 olhos de 55 pacientes considerados normais, candidatos a cirurgia refrativa no HOS (Hospital de Olhos de Sergipe) e 25 olhos de 18 pacientes portadores de ceratocone estágios 1 e 2 da classificação de Krumeich $^{(18)}$. As avaliações topográfica e paquimétrica (tomográfica) foram feitas por meio do sistema Orbscan II (Bausch \& Lomb), utilizando o fator acústico de 0,92. O sistema Orbscan II combina os discos de Placido com o escaneamento da luz em fenda horizontal. Todos os exames foram realizados entre abril de 2001 e novembro de 2003.

Inicialmente o ponto mais fino da córnea foi localizado automaticamente pelo aparelho. No mapa impresso, foram realizadas circunferências de raios crescentes de $1 \mathrm{~mm}$ a $7 \mathrm{~mm}$ concêntricas no ponto mais fino da córnea. Todos os pontos de cada circunferência foram anotados e digitados numa planilha desenhada no Excell (Microsoft Office XP) e no programa SPSS. No raio de $1 \mathrm{~mm}$ foram anotados 8 pontos, no de $2 \mathrm{~mm}$ 16 pontos, no de $3 \mathrm{~mm} 20$ pontos, no de $4 \mathrm{~mm} 28$ pontos, no de $5 \mathrm{~mm} 36$ pontos, no de $6 \mathrm{~mm} 40$ pontos e no de $7 \mathrm{~mm} 48$ pontos. Em seguida, foi realizada a média aritmética de todos os pontos de cada circunferência.

Os dados foram analisados pelo software SPSS para estudos estatísticos.

\section{RESULTADOS}

Dos 55 pacientes normais, 24 eram do sexo feminino $(43,63 \%)$ e 31 do sexo masculino (56,37\%). Dos 18 pacientes portadores de ceratocone, 7 eram do sexo feminino $(38,80 \%) \mathrm{e}$ 11 eram do sexo masculino $(61,20 \%)$.

A média dos valores no ponto mais fino dos pacientes normais foi igual a 511,60 $\mu \mathrm{m}$, com desvio padrão de $30,60 \mu \mathrm{m}$. A média dos valores do ponto mais fino dos pacientes portadores de ceratocone foi $424,48 \mu \mathrm{m}$, com desvio padrão de $56,57 \mu \mathrm{m}$. As 
médias dos valores médios de cada circunferência e do desvio padrão dos pacientes normais e dos pacientes portadores de ceratocone estão listadas na tabela 1 .

Utilizou-se como teste estatístico o t Student para comparar as médias das medidas paquimétricas a cada milímetro nos casos de olhos normais e nos de ceratocone, foi elaborado um gráfico de Box-Plot para visualizar estas comparações (Gráfico 1).

Pode-se observar no gráfico e tabela 1 que os valores da espessura dos olhos normais são maiores que os dos olhos com ceratocone para cada ponto de corte referente às circunferências de raio em milímetros. Estatisticamente são diferentes em cada ponto de corte $(\mathrm{p}<0,05)$.

Ainda pelo gráfico e tabela 1 observa-se que os olhos com ceratocone apresentam valores paquimétricos mais heterogêneos em cada ponto de corte que os olhos normais.

Foi determinado o porcentual da progressão da espessura corneana de cada milímetro em relação ao ponto mais fino (Gráfico 2).

\begin{tabular}{|llcccc|}
\hline \multicolumn{2}{|c}{ Tabela 1. Média e desvio padrão dos valores paquimérticos } \\
Olhos & \multicolumn{2}{c}{ Normal } & & \multicolumn{2}{c|}{ Ceratocone } \\
\cline { 2 - 3 } \cline { 5 - 6 } Ponto fino & 511,60 & 30,60 & & 424,48 & 56,57 \\
$1 \mathrm{~mm}$ & 516,05 & 30,47 & & 432,55 & 56,28 \\
$2 \mathrm{~mm}$ & 527,14 & 30,05 & & 454,26 & 55,21 \\
$3 \mathrm{~mm}$ & 538,80 & 29,78 & & 475,40 & 54,22 \\
$4 \mathrm{~mm}$ & 559,79 & 29,22 & & 511,74 & 52,89 \\
$5 \mathrm{~mm}$ & 579,74 & 29,29 & & 542,99 & 52,17 \\
$6 \mathrm{~mm}$ & 597,69 & 29,43 & & 568,92 & 50,74 \\
$7 \mathrm{~mm}$ & 625,36 & 29,63 & 601,72 & 47,79 \\
\hline
\end{tabular}

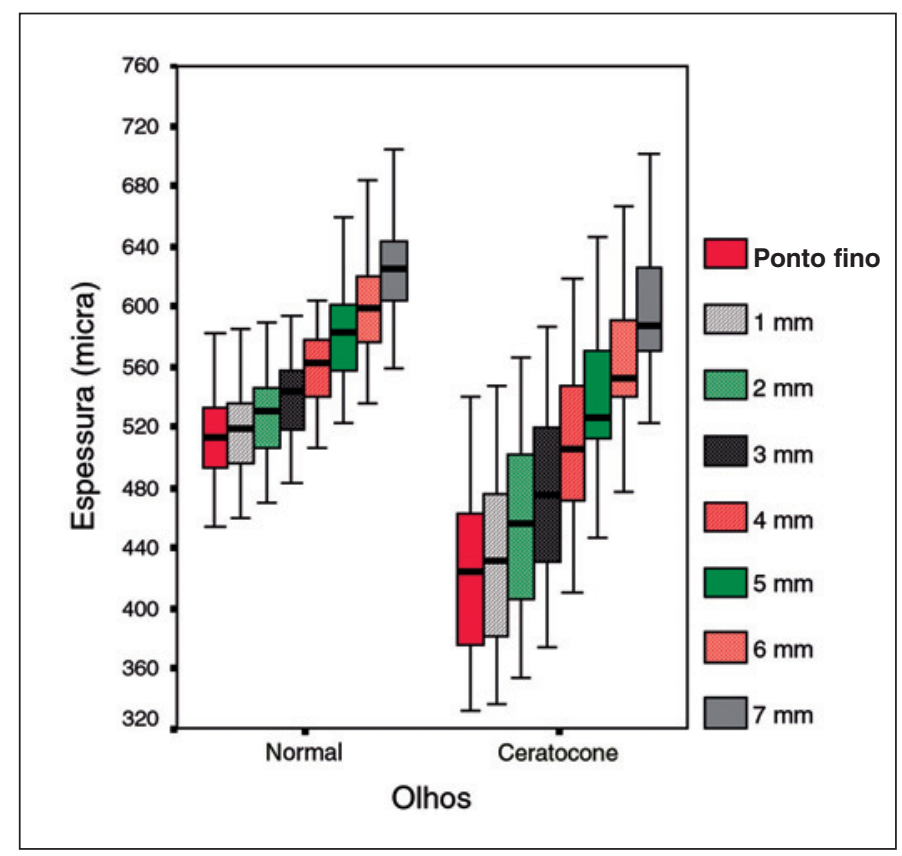

Gráfico 1 - Apresentação em Box-Plot dos valores paquimétricos em cada milímetro
$\mathrm{Na}$ tabela 2, consideramos dois níveis de significância. Apenas quando o nível de significância é determinado com $\mathrm{p}=0,01$ é que existe diferença não significativa entre a espessura dos olhos normais e dos com ceratocone nas circunferências a $6 \mathrm{~mm}$ e $7 \mathrm{~mm}$ de raio.

Segundo o gráfico 2, observamos que a progressão porcentual da espessura dos olhos com ceratocone é maior que a progressão porcentual da espessura dos olhos normais. No ponto de corte $7 \mathrm{~mm}$ obtemos a máxima progressão porcentual média de $41,8 \%$ nos olhos com ceratocone comparada com a progressão porcentual média nos olhos normais de $22,2 \%$. No caso da mínima progressão, ela acontece no ponto de corte $1 \mathrm{~mm}$, sendo $1,9 \%$ nos olhos com ceratocone e $0,9 \%$ nos olhos normais.

Observa-se no gráfico 3 a progressão porcentual individual dos 25 casos de ceratocone (indicados em vermelho) e de dois casos (indicados em verde) entre os 100 de olhos normais com curvas que indicam os extremos de progressão porcentual, ou seja, dentre os olhos normais àqueles que obtiveram a maior e menor progressão porcentual.

\begin{tabular}{|c|c|c|c|c|}
\hline & \multicolumn{4}{|c|}{ t-test para igualdade de médias } \\
\hline & $t$ & p valor & $p=0,05$ & $p=0,01$ \\
\hline Ponto fino & 7,432838 & 0,00000004682 & $\mathrm{~s}$ & $s$ \\
\hline $1 \mathrm{~mm}$ & 7,159800 & 0,00000009336 & s & s \\
\hline $2 \mathrm{~mm}$ & 6,367829 & 0,00000072443 & s & s \\
\hline $3 \mathrm{~mm}$ & 5,636491 & 0,00000506197 & s & s \\
\hline $4 \mathrm{~mm}$ & 4,378573 & 0,00015351969 & s & s \\
\hline $5 \mathrm{~mm}$ & 3,390230 & 0,00210193837 & s & s \\
\hline $6 \mathrm{~mm}$ & 2,722705 & 0,01098904657 & s & ns \\
\hline $7 \mathrm{~mm}$ & 2,361780 & 0,02517376195 & s & ns \\
\hline
\end{tabular}

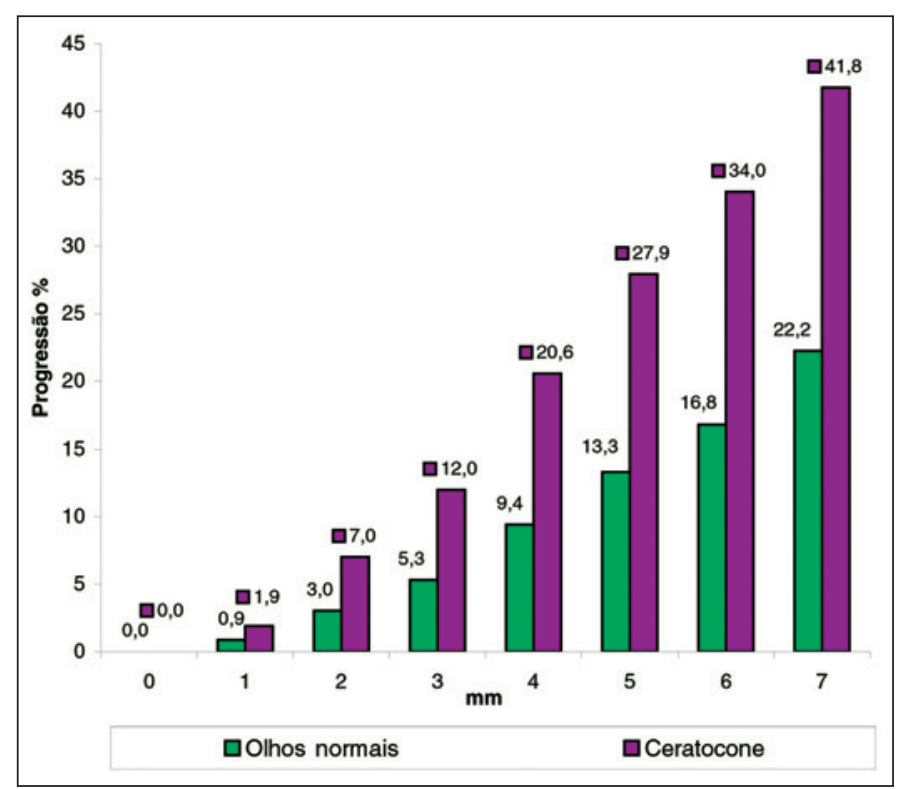

Gráfico 2 - Progressão percentual das médias dos valores paquimétricos 


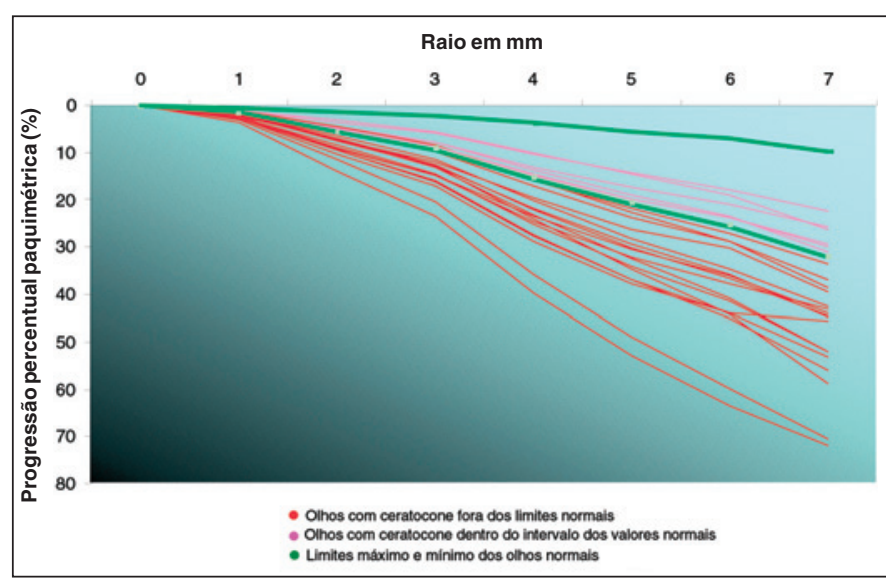

Gráfico 3 - Progressão porcentual individual

Quanto mais afastado do ponto mais fino, maior a progressão da espessura corneana nos dois grupos, todavia, esta progressão é mais acentuada nos olhos com ceratocone.

\section{DISCUSS ÃO}

Nesse presente estudo, testamos se a progressão dos valores de espessura corneana representa uma característica da arquitetura corneana de diferenciação de córneas normais de córneas com ceratocone. A hipótese surgiu de observações de Maurice, em que os raios de curvatura anterior e posterior da córnea são distintos, havendo uma espessura menor na região central e um aumento de espessura fisiológico em direção à periferia ${ }^{(19)}$. Quando a hipótese fora aventada, desconhecíamos os relatos de Mandell da variação espacial da espessura corneana como um teste diagnóstico ${ }^{(20)}$. Em 1969, Mandell, por meio de paquimetria óptica não computadorizada ao longo do meridiano horizontal, gerou curvas de progressão em direção às regiões nasal e temporal em olhos normais e com ceratocone, encontrando diferenças significantes. Certamente a complexidade e baixa reprodução das medidas impediram que esse método fosse difundido.

Considerando os resultados obtidos no presente estudo, da progressão paquimétrica, serem estatisticamente diferentes em todas as posições em olhos normais e com ceratocone, observamos que parâmetros e índices obtidos com a tomografia corneana tenham grande potencial para diagnóstico e prognóstico.

A tomografia de córnea representa um verdadeiro avanço da medida da espessura central pontual com o ultra-som e complementa significativamente as informações da topografia corneana de superfície (Placido). O sistema de escaneamento em fenda horizontal do Orbscan foi o primeiro disponível comercialmente e gera um mapa paquimétrico, além de mapas de elevação anterior e posterior e mapas de curvatura. É opinião dos autores que o mapa paquimétrico traz as informações mais relevantes para a avaliação da arquitetura tecidual. O mapa paquimétrico considera os dados da córnea posterior e anterior em conjunto, não necessitando de uma superfície de referência, como os mapas de elevação. Entretanto, essa avaliação perde confiabilidade nas córneas com algum grau de comprometimento da transparência, como as com nébulas ou leucomas, as já operadas por cirurgias refrativas e após ceratoplastia. Excetuando tais casos, a paquimetria óptica de toda a córnea com identificação do ponto mais fino é confiável e útil no diagnóstico das ectasias corneanas, bem como na avaliação pré-operatória de pacientes candidatos para cirurgia refrativa. Um fator de correção de 0,92 é preconizado para correlação com os dados obtidos com o ultra-som. Devemos considerar que o mapa de curvatura (ceratométrico) obtido pelo sistema de escaneamento em fenda é de qualidade inferior a do topógrafo de superfície por discos de Placido com, por exemplo, 16 mil pixels. Com isso pode-se colocar que ambos os sistemas sejam complementares, pois o topógrafo não permite avaliação da espessura. Nesse sentido, foi introduzido o sistema híbrido do Orbscan II que traz um topógrafo com discos de Placido acoplado ao sistema de escaneamento em fenda. Novas tecnologias vêm sendo introduzidas como o ultra-som de altíssima frequiência (Ártemis, Ultralink), a tomografia de coerência óptica de segmento anterior (Visante, Zeiss) e o Pentacam (Oculus), que utiliza um sistema de rotação em $360^{\circ}$ de uma câmera que utiliza os princípios fotográficos de Scheimpflug. Considerando os dados deste estudo e outros semelhantes realizados no Pentacam, o sistema Pentacam tem disponível um software que integra as informações do mapa paquimétrico de forma automática, de forma semelhante à realizada nesse estudo. Acreditamos que esse seja um verdadeiro avanço no software para colaborar clinicamente na avaliação dos pacientes candidatos para cirurgia refrativa ou em acompanhamento clínico de doenças ectásicas. Um sistema promissor, que combina os princípios de rotação com duas câmeras e os discos de Placido, foi introduzido pela Ziemer (Galilei), mas ainda não está disponível clinicamente.

A biomecânica do tecido corneano está relacionada à sua espessura, mas não apenas com um ponto, mas com a distribuição da espessura em toda sua extensão. Avaliar este aspecto é um dos temas com maior interesse e investimento na Oftalmologia. Vem enriquecendo não só o estudo do paciente candidato a cirurgia refrativa como também a avaliação do paciente portador ou sob suspeita de glaucoma. Recentemente o OHTS (Ocular Hypertensive Treatment Study) identificou os resultados obtidos através da paquimetria ultra-sônica como um dos fatores de risco mais importantes para o desenvolvimento de lesão glaucomatosa em paciente com hipertensão ocular ${ }^{(21)}$ Entretanto, a resistência do tecido corneano é um fator mais complexo do que a simples variação de sua espessura. Estudos utilizando o sistema de tonometria de não contato (ORA - Ocular Response Analyzer, Reichert) que gera informação da histerésis corneana, mostram que dois pacientes podem apresentar a mesma espessura de córnea, mas com características viscoelásticas diferentes e, com isso, 
diferentes influências na medida da PIO (Pressão intra-ocular $)^{(21)}$. Os dados fornecidos pela curva de normalidade da progressão dos valores paquimétricos e o cálculo de índices de progressão paquimétrica (tomográfica) podem representar indiretamente a biomecância do tecido corneano, mas essa hipótese necessita de mais estudos futuros.

\section{CONCLUSÃO}

A progressão dos valores paquimétricos é diferente em olhos normais e com ceratocone leve, sendo um parâmetro que pode ser explorado clinicamente para determinar diagnóstico possivelmente mais precoce de ectasias, bem como o prognóstico de cirurgias refrativas. Além disso, a determinação do real ponto mais fino da córnea e sua localização supera as limitações relacionadas à propedêutica tradicional da paquimetria pontual central devendo, portanto, a tomografia de córnea ser considerada no pré-operatório de cirurgias refrativas.

\section{AGRADECIMENTOS}

Agradecemos ao Dr. Carlos Arce (Campinas) pelos comentários sobre o estudo e, principalmente, por nos chamar a atenção ao estudo de Mandell (Arch Ophthalmol. 1969) ${ }^{20}$. Agradecemos ao estatístico Daniel Castañeda (Professor de estatística da Universidade Federal de Sergipe).

\section{ABSTRACT}

Purpose: To study the variation and progression of the pachymetric values from the thinnest point towards the limbus in normal and keratoconic corneas; to establish reference curves for this parameter. Methods: One hundred eyes with normal corneas and twenty-five eyes with mild keratoconus (stages I and II - Krumeich) were analyzed using the Orbscan. Concentric circles were drawn on the thinnest point of the cornea with increasing radii from 1 to $7 \mathrm{~mm}$. The average results of each circle were calculated and inserted in an Excel table in order to arrange a progression chart for each case starting on the thinnest point. The SPSS software was also used for statistical analysis. Student's t test was then used to compare the found values. Results: The average values on the thinnest point in normal patients was $511.6 \mu \mathrm{m}$ (standard deviation 30.6). The average values of the thinnest point in patients with keratoconus was $424.4 \mu \mathrm{m}$ (standard deviation 56.57). Statistically significant different values were reported $(\mathrm{p}<0.01)$ for all circles, and for 6 and $7 \mathrm{~mm}$ radii $\mathrm{p}=0.01$. Conclusion: There is a greater pachymetric variability in patients with keratoconus. Keratoconic corneas also have a faster progression of pachymetric values than healthy eyes. Pachymetric progression complements traditional single point evaluation of corneal thickness and must be considered for the screening at refractive candidates. This parameter may represent an indirect index of the biomechanics of corneal tissue but this hypothesis still needs further studies.

Keywords: Cornea, Corneal diseases, Keratoconus, Keratomileusis, laser in situ; Corneal topography; Reference values

\section{REFERÊNCIAS}

1. Ambrosio R Jr, Klyce SD, Wilson SE. Corneal topographic and pachymetric screening of keratorefractive patients. J Refract Surg. 2003;19(1):24-9.

2. Ambrósio, R Jr. Resistência corneana: variável fundamental em glaucoma e cirurgia refrativa. Rev Oftalmol Foco. 2003;86:14-6.

3. Rodrigues MM, Newsome DA, Krachmer JH, Eiferman RA. Pellucid marginal corneal degeneration: a clinicopathologic study of two cases. Exp Eye Res. 1981;33(3):277-88.

4. Andreassen TT, Simonsen AH, Oxlund H. Biomechanical properties of keratoconus and normal corneas. Exp Eye Res. 1980;31(4):435-41.

5. Barraquer JI. Keratomileusis for myopia and aphakia. Ophthalmology. 1981;88 (8):701-8.

6. Barraquer JI. Basis of refractive keratoplasty-1967. Refract Corneal Surg. 1989; 5(3):179-93.

7. Price FW Jr, Koller DL, Price MO. Central corneal pachymetry in patients undergoing laser in situ keratomileusis. Ophthalmology. 1999;106(11):221620. Comment in: Ophthalmology. 2000;107(11):1967-8.

8. Seiler T, Quurke AW. Iatrogenic keratectasia after LASIK in a case of forme fruste keratoconus. J Cataract Refract Surg. 19981;24(7):1007-9. Comment in: J Cataract Refract Surg. 2000;26(5):629-30.

9. Amoils SP, Deist MB, Gous P, Amoils PM. Iatrogenic keratectasia after laser in situ keratomileusis for less than -4.0 to -7.0 diopters of myopia. J Cataract Refract Surg. 2000;26(7):967-77. Comment in: J Cataract Refract Surg. 2001; 27(4):490-1. J Cataract Refract Surg. 2001;27(1):4-6; author reply 6-7. J Cataract Refract Surg. 2001;27(1):4; author reply 6-7; J Cataract Refract Surg. 2001; 27(1):4; author reply 6-7.

10. Rao SN, Epstein RJ. Early onset ectasia following laser in situ keratomileusis: case report and literature review. J Refract Surg. 2002;18(2):177-84.

11. Schmitt-Bernard CF, Lesage C, Arnaud B. Keratectasia induced by laser in situ keratomileusis in keratoconus. J Refract Surg. 2000;16(3):368-70.

12. McLeod SD, Kisla TA, Caro NC, McMahon TT. Iatrogenic keratoconus: corneal ectasia following laser in situ keratomileusis for myopia. Arch Ophthalmol. 2000;118(2):282-4.

13. Jabbur NS, Stark WJ, Green WR. Corneal ectasia after laser-assisted in situ keratomileusis. Arch Ophthalmol. 2001;119(11):1714-6.

14. Lafond G, Bazin R, Lajoie C. Bilateral severe keratoconus after laser in situ keratomileusis in a patient with forme fruste keratoconus. J Cataract Refract Surg. 2001;27(7):1115-8.

15. Seiler T, Koufala K, Richter G. Iatrogenic keratectasia after laser in situ keratomileusis. J Refract Surg. 1998;14(3):312-7.

16. Geggel HS, Talley AR. Delayed onset keratectasia following laser in situ keratomileusis. J Cataract Refract Surg. 1999;25(4):582-6. Comment in: J Cataract Refract Surg. 1999;25(4):453-4. J Cataract Refract Surg. 1999;25(8):1036-7.

17. Holland SP, Srivannaboon S, Reinstein DZ. Avoiding serious corneal complications of laser assisted in situ keratomileusis and photorefractive keratectomy. Ophthalmology. 2000;107(4):640-52. Comment in: Ophthalmology. 2001;108 (5):834-5.

18. Krumeich JH, Daniel J, Knülle A. Live-epikeratophakia for keratoconus. J Cataract Refract Surg. 1998;24(4):456-63.

19. Maurice DM. The cornea and sclera. In: Davson H, editor. The eye: vegetative physiology and biochemistry. 3 ed. Orlando: Academic Press; 1984.

20. Mandell RB, Polse KA. Keratoconus: spatial variation of corneal thickenss as a diagnostic test. Arch Ophthalmol. 1969;82(2):182-8.

21. Brandt JD, Beiser JA, Kass MA, Gordon MO. Central corneal thickness in the Ocular Hypertension Treatment Study (OHTS). Ophthalmology. 2001;108(10): 1779-88. 